\title{
Three-nucleon system: Irreducible and reducible contributions of the three-nucleon force
}

\author{
A. Deltuva \\ Institute of Theoretical Physics and Astronomy, Vilnius University, A. Goštauto 12, LT-01108 Vilnius, Lithuania \\ P. U. Sauer \\ Institute for Theoretical Physics, Leibniz University Hannover, D-30167 Hannover, Germany \\ (Received 15 January 2015; revised manuscript received 1 March 2015; published 16 March 2015)
}

\begin{abstract}
The three-nucleon bound and scattering equations are solved in momentum space for a coupled-channels Hamiltonian. The Hamiltonian couples the purely nucleonic sector of Hilbert space with a sector in which one nucleon is excited to a $\Delta$ isobar. The interaction consists of irreducible two-baryon and irreducible three-baryon potentials. The calculation keeps only the purely nucleonic one among the irreducible three-baryon potentials. The coupled-channels two-baryon potential yields additional reducible contributions to the three-nucleon force. The Coulomb interaction between the two protons is included using the method of screening and renormalization. Three-nucleon force effects on the bound-state energies and on observables of elastic nucleon-deuteron scattering and breakup are studied.
\end{abstract}

DOI: 10.1103/PhysRevC.91.034002

PACS number(s): 21.30.-x, 21.45.-v, 24.70.+s, 25.10.+s

\section{MOTIVATION}

The notion of the three-nucleon $(3 N)$ force is not a uniquely defined concept for the theoretical description of nuclear phenomena. The $3 N$ force arises in the hadronic picture of nuclear systems, a model developed by theoreticians for calculational convenience. The $3 N$ force is therefore also model dependent and experimentally not measurable.

The microscopic degrees of freedom underlying nuclear phenomena are the quarks and gluons of quantum chromodynamics (QCD). However, a direct description of nuclear phenomena in terms of those microscopic degrees of freedom is not available yet; instead, the effective description in terms of quark-gluon clusters, i.e., in terms of hadrons and their interactions among each other and with electroweak probes is a common conceptual and often quantitatively successful approach to nuclear phenomena at low and moderate energies which we also adopt. At low energies, rigid nucleons appear to make up nuclei in bound and scattering states; after all, nuclear bound states have masses which are almost multiples of the one of a single nucleon. At energies above the pion$(\pi)$ production threshold, the $\Delta$ isobar and the $\pi$ meson become additional active degrees of freedom. The interactions between the hadronic constituents of nuclear systems depend on the chosen degrees of freedom, i.e., on the energy range of applicability; they are mediated by exchanged mesons. The dynamics is usually assumed to be controlled by a field theory for the effective hadronic degrees of freedom, originally by a phenomenological field theory with a zoo of mesons [1]. At present, the favorably employed one is the chiral effective field theory $(\chi$ EFT) [2-4], which respects the chiral symmetries of QCD and works with the nucleon and $\pi$ as degrees of freedom, sometimes extended by the inclusion of the $\Delta$ isobar $[5,6]$.

However, for practical calculations of nuclear systems, the dynamics is chosen to follow quantum mechanics. The forces between the hadrons, arising from field-theoretic processes, have therefore to be cast into the form of Hermitian potentials. That dynamic simplification is achieved by freezing some of the field-theoretic degrees of freedom. The quantum-mechanical kinematics is nonrelativistic for the nucleon and the $\Delta$ isobar and relativistic for the $\pi$ if quantummechanically active. Quantum mechanics is advantageous, since it allows the step from two-particle to many-particle systems in a natural way. The potentials of the baryons are of two-, three-, and, in general, of $A$-particle nature, $A$ being the number of baryons making up the nuclear system under study. It is well established that at normal densities the many- $N$ potentials show a hierarchy of importance: The $2 \mathrm{~N}$ part is most important and makes the contact with the deuteron and with free two-nucleon scattering. The $3 N$ part is the first important dynamic correction of the $2 N$ interaction, whereas many- $N$ interactions are more complex than the $3 \mathrm{~N}$ one and also appear dynamically rather unimportant at normal nuclear densities. Furthermore, many-baryon contributions to the interaction, e.g., those arising from non-nucleonic baryons as the $\Delta$ isobar, are also dynamically strongly suppressed due to their reduced weights in the nuclear wave functions. That is the reason why the present paper focuses on the $3 N$ force in the hadronic picture of nuclear phenomena.

The present paper makes the following choice for the description of nuclear phenomena. The only active degrees of freedom are the nucleon and the $\Delta$ isobar, and all mesonic degrees of freedom are frozen. The Hilbert space consists of two sectors, a purely nucleonic one and a sector in which one nucleon is replaced by a $\Delta$ isobar. The Hilbert space is chosen with a view on the energy domain above the $\pi$-production threshold. The $\Delta$ isobar is an important mechanism for $\pi$ production, but since $2 \pi$ and $3 \pi$ production are strongly suppressed far above their thresholds, configurations with more than one $\Delta$ isobar should be physically less important in the energy regime up to about $0.5 \mathrm{GeV}$ excitation in the center-of-mass (c.m.) system. Our goal is the description of nuclear phenomena for energies below the $\pi$-production threshold. Though the $\pi$ is energetically not an active degree of freedom yet, the $\Delta$ isobar is already expected to make important contributions to the nuclear dynamics. That will indeed be the case, and that is the reason for the chosen 

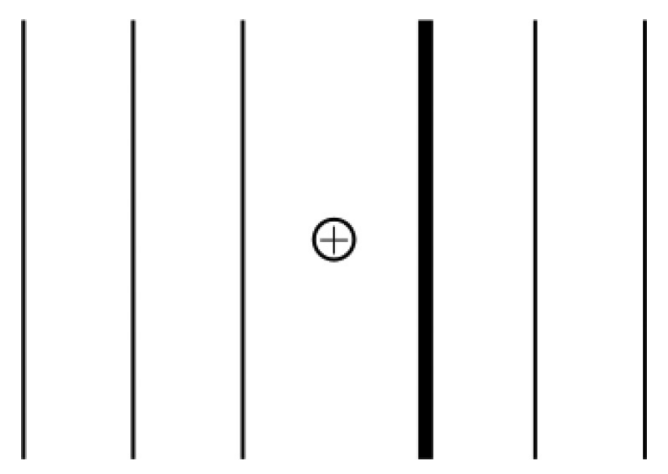

$\Delta$

FIG. 1. Hilbert space for the description of nuclear phenomena at energies below the $\pi$-production threshold. The shown example is for baryon number 3. Compared with a purely nucleonic Hilbert space, it is extended by a sector, in which one nucleon (thin line) is turned into a $\Delta$ isobar (thick line). The deuteron and two-nucleon reactions are described in the corresponding Hilbert space of baryon number 2 .

extended Hilbert space. The chosen Hilbert space is shown in Fig. 1 for the example of a three-baryon system. In the presently chosen absence of a coupling to $\pi$-nucleon states the $\Delta$ isobar is a stable baryon; its rest mass is taken to be $1.232 \mathrm{GeV}$, the resonance position of $\pi$-nucleon scattering in the $P_{33}$ partial wave.

The potentials, required for the dynamics in the chosen Hilbert space, are illustrated in Fig. 2; they are irreducible and Hermitian. The interaction Hamiltonian is of coupledchannels character: It consists of two-baryon parts which act in both sectors of the Hilbert space and which couple them by transition potentials. The Hamiltonian also contains
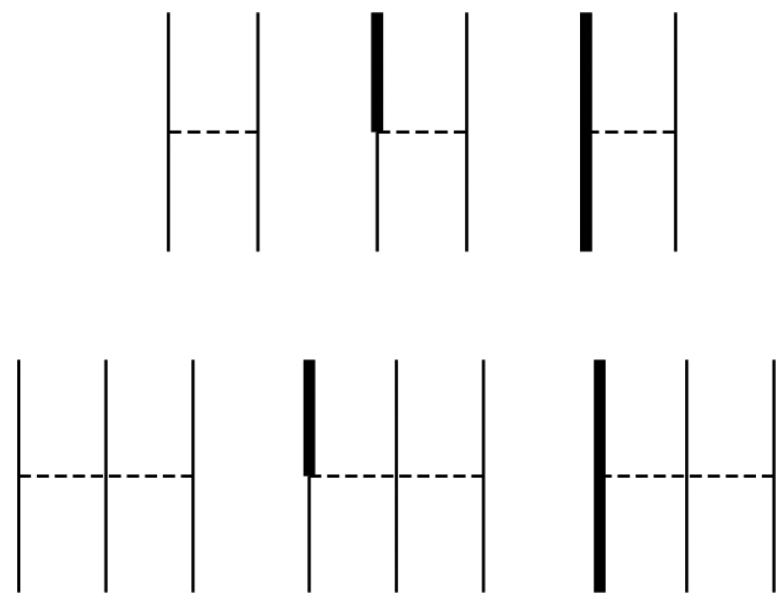

FIG. 2. Interactions describing the nuclear dynamics in the Hilbert space of Fig. 1. They are irreducible coupled-channels potentials of two-baryon (upper row) and three-baryon (lower row) nature. Besides their purely nucleonic parts, they couple the purely nucleonic sector with the one containing a $\Delta$ isobar, and they act directly in the sector containing a $\Delta$ isobar. Only selected examples of the potentials are shown; the Hermitian-conjugate pieces have to be added as well as those obtained by permuting the $\Delta$ isobar in the initial and final states to other positions in the diagrams.
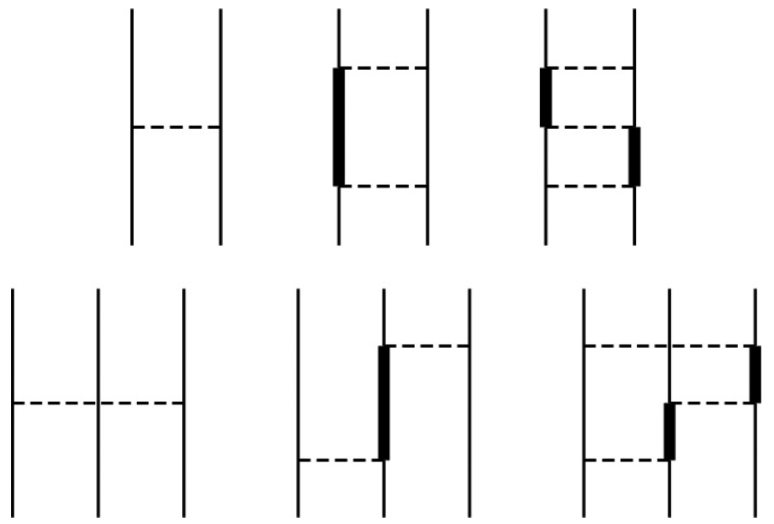

FIG. 3. Irreducible contributions and selected examples of reducible contributions to the $2 N$ (upper row) and to the $3 N$ (lower row) interactions. The two reducible $3 N$ processes shown are, in sequence, the Fujita-Miyazawa and the $\Delta$-ring processes.

three-baryon parts which also act in both sectors of the Hilbert space and couple them by transition potentials. In addition, there are more complex many-baryon potentials involving more than three baryons, up to baryon number $A$, the baryon number of the nuclear system under study. However, we shall constrain our calculations to the three-nucleon system; thus, four-baryon forces and forces of even higher complexity do not occur.

When employing the coupled-channels Hamiltonian with an explicit $\Delta$ isobar, illustrated in Fig. 2, the nuclear dynamics receives the irreducible contributions of the chosen Hamiltonian but also reducible contributions to the $2 \mathrm{~N}$ and $3 N$ interactions arising from its iterative application in the process of solving the many-nucleon problem. The interplay between irreducible and reducible contributions to the $2 N$ and $3 N$ interactions is shown in Fig. 3. Some dynamic processes, in other approaches described by irreducible potentials, are already accounted for by the coupled-channels two-baryon part of the Hamiltonian as reducible processes in the Hilbert space of Fig. 1; they have therefore to be excluded from the irreducible parts of the Hamiltonian.

The $2 N$ interaction receives an important contribution from the virtual excitation of a nucleon to the $\Delta$ isobar contributing to the attraction at intermediate range. For example, the potential CD Bonn $+\Delta$, designed by us as such a coupled-channels potential in Ref. [7] and employed by us for the description of few-nucleon systems previously, artfully takes out the virtual excitation of the $\Delta$ isobar from its irreducible part. The reducible, usually attractive contributions, illustrated in the upper row of Fig. 3, to the $2 \mathrm{~N}$ interaction get weakened in the nuclear medium, a well-known effect, called $2 N$ dispersion.

In the same way, the three-nucleon processes provided by the coupled-channels potential in a reduced form will have to be excluded from the employed irreducible $3 N$ potential. The physically most important three-nucleon processes which the coupled-channels potential already provides are the FujitaMiyazawa [8] and the $\Delta$-ring processes, as illustrated in the lower row of Fig. 3; the importance of those processes is seen in our own calculations, but it is also confirmed in the context of other dynamic strategies in Refs. [9,10]. 
The paper studies the three-nucleon system. Its objective is twofold: It has a physics aspect and a technical aspect:

(i) The paper extends the dynamics of our previous calculations [11,12] by adding an irreducible $3 N$ force to the employed coupled-channels two-baryon potential. Our previous calculations without such an irreducible $3 N$ force are physically incomplete and missed the three-nucleon binding energies and the neutron-deuteron doublet scattering length. We would like to improve on both quantities with the physics question: Is their simultaneous improvement possible? How serious is the miss on those two observables for the prediction of three-nucleon scattering at higher energies?

(ii) The paper demonstrates that complete and reliable calculations including reducible and irreducible $3 N$ forces can be done for the bound and scattering states of the three-nucleon system. As will be discussed in Sec. II B, the chosen dynamics is not yet in all its respects up to the most modern standards. But the paper paves the way to further, dynamically more satisfactory, calculations. That is the technical objective of this paper.

\section{CALCULATiONAL APPARATUS}

The paper calculates the three-nucleon bound and scattering states, i.e., nucleon-deuteron elastic scattering and breakup, for energies up to the $\pi$-production threshold. Those calculational goals require the extension of two numerical schemes, used previously by the authors. Our standard approach to the three-nucleon system is based on a coupled-channels twobaryon potential as in Refs. [11,12]; note that Ref. [12] and all our recent calculations overcame the need for a separable expansion of the two-baryon transition matrix, still required in Ref. [11]. In contrast, Ref. [13] uses irreducible two- and three-baryon forces for the three-nucleon bound states and scattering, but the forces are purely nucleonic ones. The present paper requires the combination of those two schemes. Reference [14] already implemented such a combination of a coupled-channels two-baryon potential with an explicit $\Delta$ isobar and of an additional irreducible $3 N$ force, but the application was constrained to the three-nucleon bound state; the present paper extends that approach to the three-nucleon scattering. Furthermore, the coupled-channels potential, employed in Ref. [14], was not properly fitted to the two-nucleon data.

\section{A. The equations}

There are different strategies for setting up the quantummechanical equations for obtaining the three-nucleon bound and scattering states. We work in momentum-space representation and employ integral equations of the Alt, Grassberger, and Sandhas (AGS) type [15]. There are three pairwise potentials $v_{\alpha}$, i.e.,

$$
V_{(2)}=\sum_{\alpha=1}^{3} v_{\alpha},
$$

$\alpha$ being the label of the noninteracting particle during the pairwise interaction. Each of the three potentials is summed up into the respective two-particle transition matrix $T_{\alpha}(z)$, i.e.,

$$
T_{\alpha}(z)=v_{\alpha}+v_{\alpha} G_{0}(z) T_{\alpha}(z)
$$

$G_{0}(z)=\left(z-H_{0}\right)^{-1}$ is the free three-particle resolvent with the available energy $z$ and the free Hamiltonian $H_{0}$ containing the kinetic energy of the particles and their rest mass differences in the three-nucleon system with $\Delta$-isobar states the difference with respect to three nucleons. The three-particle potential

$$
V_{(3)}=\sum_{\gamma=1}^{3} u_{\gamma}
$$

is also decomposed into three terms $u_{\gamma}$ that are symmetric under the exchange of particles $\beta \neq \gamma$ and $\alpha \neq \gamma$ and can be transformed into one another by cyclic permutations. The decomposition (3) is not unique. Often the $3 N$ force is written such that $\gamma$ labels the particle which is directly linked to the two others in the interaction [16]. However, such a decomposition is inefficient for our practical calculation, and we will therefore follow the procedure of Refs. [13,17]; this procedure for the decomposition gets explained in Sec. II B.

The strategy for setting up the integral equations works with the two-particle part of the potential summed into the transition matrix $T_{\alpha}(z)$, but keeping the three-particle part in its potential form $V_{(3)}$ uniterated. The basis of states, employed for calculations in the three-nucleon system, is, in the purely nucleonic Hilbert sector, the direct product of an antisymmetrized nucleon pair and a third nucleon without antisymmetrization; thus, the basis is overcomplete. In the Hilbert sector with a $\Delta$ isobar the same pair symmetrization is used, since the $\Delta$ isobar can be created from any of the three nucleons in a symmetric fashion. The isospin formalism is used for distinguishing protons and neutrons including both total isospin $\frac{1}{2}$ and $\frac{3}{2}$ states. The results of the integral equations are bound-state amplitudes $\left|\psi_{\alpha}\right\rangle$ and multichannel transition amplitudes $U_{\beta \alpha}$, connecting the initial two-body channel with the noninteracting particle of label $\alpha$ to the final two-body channel with the noninteracting particle of label $\beta, \beta=0$ being the final three-particle breakup channel. All multichannel transition amplitudes depend on the available energy $E+i 0$; that dependence is notationally suppressed. The derivation of the equations is given in Ref. [13]; the fact, that Ref. [13] describes the three-nucleon system in terms of a completely nucleonic Hilbert space is immaterial for the derivation of equations. The resulting equations of Ref. [13] can therefore be taken over without any change.

\section{Bound state}

The three-particle bound-state wave function

$$
|\Psi\rangle=\sum_{\alpha}\left|\psi_{\alpha}\right\rangle
$$

is decomposed into its Faddeev components $\left|\psi_{\alpha}\right\rangle$. Given the identity of nucleons, carried over to states with a $\Delta$ isobar, the three Faddeev components are related by the permutation 
of the baryons. Any Faddeev component can be taken as a symmetrized Faddeev amplitude $|\psi\rangle$ which is obtained from

$$
|\psi\rangle=G_{0} T P|\psi\rangle+\left(1+G_{0} T\right) G_{0} u(1+P)|\psi\rangle,
$$

yielding the bound-state wave function in the form $|\Psi\rangle=$ $(1+P)|\psi\rangle$ with the help of the permutation operator $P$, i.e., $P=P_{12} P_{23}+P_{13} P_{23}, P_{\beta \alpha}$ being the individual permutation operator of baryons $\beta$ and $\alpha$.

\section{Scattering operators}

The multichannel transition amplitudes $U_{\beta \alpha}$ act on the two-body channel states $\left|\phi_{\alpha}\right\rangle$, the eigenstates of the corresponding channel Hamiltonian $H_{\alpha}=H_{0}+v_{\alpha}$ with the energy eigenvalue $E$. Due to symmetry, all three channel states are related to each other by the permutation $P$, defined in the previous subsection. One channel therefore can be taken as characteristic for all. Its channel label will therefore be dropped as well as the label for the component $u_{\gamma}$ of the three-nucleon potential, and one can work with a symmetrized transition operator $U$ for elastic scattering, obtained from the symmetrized AGS integral equation, i.e.,

$$
\begin{aligned}
U= & P G_{0}^{-1}+(1+P) u+P T G_{0} U \\
& +(1+P) u G_{0}\left(1+T G_{0}\right) U,
\end{aligned}
$$

yielding the on-shell matrix elements $\langle\phi|U| \phi\rangle$, required for the calculation of observables. The breakup operator $U_{0}$, connecting an initial two-body channel with the final breakup channel $\left|\phi_{0}\right\rangle$ of noninteracting nucleons, is then obtained by quadrature,

$$
U_{0}=(1+P)\left[G_{0}^{-1}+u+T G_{0} U+u G_{0}\left(1+T G_{0}\right) U\right],
$$

yielding the on-shell matrix elements $\left\langle\phi_{0}\left|U_{0}\right| \phi\right\rangle$, required for the calculation of breakup observables.

In the following, elastic scattering observables are shown as functions of the center-of-mass scattering angle $\Theta_{\text {c.m. }}$, while breakup observables as functions of the arclength $S$ along the kinematical curve. As in Ref. [13], we obtain well-converged results by taking into account the hadronic interaction in twobaryon partial waves with the total pair angular momentum $j_{x} \leqslant 5$ and including three-baryon states with total $3 N$ angular momentum $\mathcal{J} \leqslant \frac{59}{2}$; it is fully sufficient to limit the irreducible $3 N$ force to states with $\mathcal{J} \leqslant \frac{19}{2}$. In all calculations of Sec. III B for the three-nucleon system with two protons their Coulomb repulsion is added with the necessary changes in the above equations according to Refs. [18-21].

\section{B. Choice of the dynamics}

We take over the potential CD Bonn $+\Delta$ [7] as coupledchannels two-baryon potential, keeping its parameters unchanged in this paper. It is based on the exchange of $\pi, \rho$, $\omega$, and $\sigma$ mesons. We note that this potential is tuned to the deuteron and to two-nucleon elastic scattering below 350-MeV laboratory energy and that the potential parts, connected with the $\Delta$ isobar, are therefore underdetermined by that procedure. Those potential parts would be physically better determined by a simultaneous fit to inelastic two-nucleon scattering, i.e., to the data of channels with a single $\pi$. However, at present such a tuning procedure is too demanding for us; it would also require the addition of a Hilbert sector with a single $\pi$ and the corresponding additions to the Hamiltonian part of baryon number 2.

We add an irreducible $3 N$ potential to the Hamiltonian. Among the multitude of possible three-baryon potentials, illustrated in the lower row of Fig. 2, the $3 N$ one was argued by us in Sec. I to be most important.

We choose the Urbana IX $3 N$ force [16] as starting point for our construction of $3 N$ potential models, employed in this paper. This choice of a phenomenological irreducible $3 N$ potential is against our original philosophy when developing a coupled-channels two-baryon potential: $2 N$ and $3 N$ forces have to be physically consistent with each other. The coupled-channels approach creates such a satisfying consistency between the $2 N$ and the reducible $3 N$ interactions; but this consistency is broken by the addition of a phenomenological irreducible $3 N$ potential, as done now. The Urbana IX $3 N$ force, chosen as starting point for the modeling in the present paper, has $2 \pi$-exchange and phenomenological repulsive short-range terms, i.e.,

$$
\begin{aligned}
V_{(3)}= & \sum_{\alpha \beta \gamma \text { cyclic }}\left(A_{2 \pi}\left\{X_{\alpha \beta}^{\pi}, X_{\beta \gamma}^{\pi}\right\}\left\{\tau_{\alpha} \cdot \tau_{\beta}, \tau_{\beta} \cdot \tau_{\gamma}\right\}\right. \\
& \left.+C_{2 \pi}\left[X_{\alpha \beta}^{\pi}, X_{\beta \gamma}^{\pi}\right]\left[\tau_{\alpha} \cdot \tau_{\beta}, \tau_{\beta} \cdot \tau_{\gamma}\right]+B_{0} T_{\alpha \beta}^{2} T_{\beta \gamma}^{2}\right),
\end{aligned}
$$

where curly and square brackets denote anticommutators and commutators, respectively. The strength constants were chosen in Ref. [16] to be $A_{2 \pi}=-0.0293 \mathrm{MeV}, C_{2 \pi}=\frac{1}{4} A_{2 \pi}$, and $B_{0}=0.0048 \mathrm{MeV}$. Here $\tau_{\beta}$ is the isospin operator of the nucleon $\beta$, while the potential-like pair $(\alpha \beta)$ operators $X_{\alpha \beta}^{\pi}$ and $T_{\alpha \beta}$ are given in coordinate space by

$$
\begin{aligned}
X_{\alpha \beta}^{\pi} & =Y\left(m_{\pi} r_{\alpha \beta}\right) \sigma_{\alpha} \cdot \sigma_{\beta}+T\left(m_{\pi} r_{\alpha \beta}\right) S_{\alpha \beta}, \\
T_{\alpha \beta} & =T\left(m_{\pi} r_{\alpha \beta}\right),
\end{aligned}
$$

with $\sigma_{\alpha}$ being the spin operator of the nucleon $\alpha, S_{\alpha \beta}$ the tensor operator of the pair $\alpha \beta, m_{\pi}$ the average $\pi$ mass, and $Y(x)$ and $T(x)$ the Yukawa and tensor functions specified in Ref. [22]; all operators are of $\pi$ range. In our calculation $X_{\alpha \beta}^{\pi}$ and the squared operators $T_{\alpha \beta}^{2}$ are transformed to momentum space using spherical Bessel functions. Since the decomposition of $V_{(3)}$ into three components according to Eq. (3) is not unique, we make the following choice: We expand the sum (7) and take as $u_{\gamma}$ the terms where the operator acting on the final state (the operator that is on the left side in the product) is either $X_{\alpha \beta}^{\pi}$ or $T_{\alpha \beta}^{2}$, i.e.,

$$
\begin{aligned}
u_{\gamma}= & 2 X_{\alpha \beta}^{\pi}\left\{X_{\beta \gamma}^{\pi}\left[A_{2 \pi} \tau_{\alpha} \cdot \tau_{\gamma}-i C_{2 \pi} \tau_{\alpha} \cdot\left(\tau_{\beta} \times \tau_{\gamma}\right)\right]\right. \\
& \left.+X_{\gamma \alpha}^{\pi}\left[A_{2 \pi} \tau_{\gamma} \cdot \tau_{\beta}+i C_{2 \pi} \tau_{\alpha} \cdot\left(\tau_{\beta} \times \tau_{\gamma}\right)\right]\right\} \\
& +\frac{1}{2} B_{0} T_{\alpha \beta}^{2}\left(T_{\beta \gamma}^{2}+T_{\gamma \alpha}^{2}\right) .
\end{aligned}
$$

$V_{3}$ gets then decomposed as in Eq. (3). This decomposition is more efficient in our practical calculations as argued in Refs. [13,17]. The numerical technique is taken over from Ref. [13] and adapted here for the context of additional nonnucleonic channels. This technical possibility of casting the 
$3 N$ potential $V_{3}$ into the form (3) with $u_{\gamma}$ of Eq. (9) was partly the reason for choosing the Urbana IX $3 N$ force as basis for our search of a useful irreducible $3 N$ force supplementing a given coupled-channels two-baryon potential.

The $A_{2 \pi}$ and $C_{2 \pi}$ terms in $u_{\gamma}$ and $V_{3}$ are related to $2 \pi$-meson exchange; the Fujita-Miyazawa mechanism, illustrated in its reducible form in Fig. 3, makes an important contribution to the $3 N$ potential, requiring the parameter combination $C_{2 \pi}=\frac{1}{4} A_{2 \pi}$, as chosen in Ref. [16]. Since that dynamic part is provided by the employed coupled-channels two-baryon potential CD Bonn $+\Delta$ in a reducible form, the FujitaMiyazawa contribution has to be taken out from the parameters in our reparametrization of an irreducible $3 N$ potential in the Urbana-like fashion. We note in passing that the reducible Fujita-Miyazawa process, provided by the coupled-channels two-baryon potential CD Bonn $+\Delta$, also includes the $\rho$-meson exchange and is therefore richer than the original FujitaMiyazawa $3 N$ force of Ref. [8]. Compared to the Urbana IX $3 N$ force the strength of the potential should be reduced and deviations from the ratio $C_{2 \pi} / A_{2 \pi}=\frac{1}{4}$ are acceptable. In fact, that ratio $C_{2 \pi} / A_{2 \pi}$ got increased in Ref. [14] when the Fujita-Miyazawa process was removed there from the TucsonMelbourne $3 N$ potential [23]. We therefore have to expect a similar increase of that ratio $C_{2 \pi} / A_{2 \pi}$ in our reparametrization of an irreducible $3 N$ potential in the Urbana-like fashion.

The repulsion with the strength parameter $B_{0}$ in the Urbana IX $3 N$ force was required to prevent overbinding of nuclear matter. However, that argument is not relevant in the present context any longer. The coupled-channels two-baryon potential provides repulsion due to the two-nucleon dispersion. That fact was confirmed for nuclear matter by Ref. [24]. We therefore expect that our reparametrization of the irreducible $3 N$ potential should have a smaller or even a vanishing $B_{0}$ value compared to the Urbana IX $3 N$ force. We therefore set $B_{0}=0$ in our first try and retune $A_{2 \pi}$ and $C_{2 \pi}$ having in mind that the resulting dynamic model has to account for the ${ }^{3} \mathrm{H}$ binding energy $\left|E_{3^{3} \mathrm{H}}\right|$ and attempting also to fit the neutron-deuteron doublet scattering length $a_{2}$. The potential CD Bonn $+\Delta$ misses $\left|E_{3^{H}}\right|$ only by $176 \mathrm{keV}$ and with $a_{2}=0.695 \mathrm{fm}$ is already pretty close to the experimental value of $0.65 \pm 0.04 \mathrm{fm}$ [25]. Thus, the required parameters in the added modified Urbana-like $3 N$ potential should be quite mild ones.

However, with the coupled-channels two-baryon potential $\mathrm{CD}$ Bonn $+\Delta$ and the modified Urbana IX $3 N$ force as irreducible $3 N$ force in addition, we are unable to fit the ${ }^{3} \mathrm{H}$ binding energy $\left|E_{3^{3}}\right|$ and the neutron-deuteron doublet scattering length $a_{2}$ simultaneously with weak $A_{2 \pi}$ and $C_{2 \pi}$ parameters. We therefore included also the $B_{0}$ term and tried a wide range of parameters, using a rather coarse raster but staying within sensible limits. In the end we chose two groups of modifications only, whose parameters are listed in Table I and which appear to us characteristic for our parameter search with Urbana-like $3 N$ potentials. In the first group the ratio $C_{2 \pi} / A_{2 \pi}$ was set larger than $\frac{1}{4}$ by hand and not subjected to the fit.

The first group of parametrizations consists of the $3 \mathrm{~N}$ potentials (U1, U2, U3). The two terms augmented by the parameters $A_{2 \pi}$ and $C_{2 \pi}$ both yield attraction; if repulsion
TABLE I. Parametrization of the employed irreducible $3 N$ potentials. The parameters $A_{2 \pi}, C_{2 \pi}$, and $B_{0}$ refer to the form of the $3 N$ potential of Eq. (7); they are given in units of MeV. The ${ }^{3} \mathrm{H}$ binding energy $\left|E_{3 \mathrm{H}}\right|$ is given in $\mathrm{MeV}$, its experimental value being $8.482 \mathrm{MeV}$; the neutron-deuteron doublet scattering length $a_{2}$ is given in $\mathrm{fm}$, its experimental value being $0.65 \pm 0.04 \mathrm{fm}$. In contrast to other calculations in this work, the results are obtained including $3 N$ states with the $2 N$ total angular momentum $j_{x} \leqslant 6$.

\begin{tabular}{lccccc}
\hline \hline & $A_{2 \pi}$ & $C_{2 \pi} / A_{2 \pi}$ & $B_{0}$ & $\left|E_{3_{\mathrm{H}}}\right|$ & $a_{2}$ \\
\hline CD Bonn & & & & 8.004 & 0.932 \\
CD Bonn+ $\Delta$ & & & & 8.306 & 0.695 \\
CD Bonn+ $\Delta+\mathrm{U} 1$ & -0.00266 & +0.5000 & 0 & 8.482 & 0.559 \\
CD Bonn+ $\Delta+\mathrm{U} 2$ & -0.01559 & +1.0000 & 0.00625 & 8.482 & 0.606 \\
CD Bonn+ $\Delta+\mathrm{U} 3$ & -0.02598 & +1.0000 & 0.01250 & 8.482 & 0.639 \\
CD Bonn $+\Delta+\mathrm{U} 1$ & -0.00266 & +0.5000 & 0 & 8.482 & 0.559 \\
CD Bonn $+\Delta+\mathrm{U} 4$ & +0.06500 & -0.3995 & 0 & 8.482 & 0.576 \\
CD Bonn $+\Delta+\mathrm{U} 5$ & -0.06500 & -0.3551 & 0 & 8.482 & 0.539 \\
\hline \hline
\end{tabular}

is needed, it is provided by the term with the parameter $B_{0}$. All models fit $\left|E_{3_{\mathrm{H}}}\right|$ perfectly and, except for $\mathrm{U} 3$, underpredict the neutron-deuteron doublet scattering length $a_{2}$. Running down this list of three modifications (U1, U2, $\mathrm{U} 3$ ), the individual strength parameters for attraction and repulsion become increasingly larger; when accounting for the neutron-deuteron doublet scattering length $a_{2}$ as in U3, the three parameters are individually largest. Furthermore, we are disturbed that the $A_{2 \pi}$ and $C_{2 \pi}$ parts of the $3 N$ potential U3 make a massive contribution of about $5 \mathrm{MeV}$ to the triton binding $\left|E_{{ }^{3} \mathrm{H}}\right|$, which has to be balanced by a corresponding comparable repulsion due to the $B_{0}$ term, just to squeeze out the required small additional binding of $176 \mathrm{keV}$. Thus, the simultaneous account of the ${ }^{3} \mathrm{H}$ binding $\left|E_{3^{3} \mathrm{H}}\right|$ and of the neutron-deuteron doublet scattering length $a_{2}$ appears difficult. We are only able to achieve that goal with the irreducible $3 N$ potential $\mathrm{U} 3$ of questionable parametrization, i.e., the attraction and repulsion required for balancing each other, are unnaturally large. However, this tuning difficulty appears not to be a feature characteristic for our special $3 N$ force model. Indeed, in Ref. [26] another study was performed using the purely nucleonic Argonne V18 potential [22] in combination with various $3 N$ forces; reproducing experimental values of the neutron-deuteron doublet scattering length $a_{2}$ and of $3 N$ and $4 N$ binding energies required a balance of attractive and repulsive $3 N$ force contributions, individually even stronger than in the model CD Bonn $+\Delta+\mathrm{U} 3$ of this paper. Furthermore, Ref. [27] used purely nucleonic $2 N$ and $3 N$ potentials consistently derived from $\chi \mathrm{EFT}$, thus, it uses rather different dynamics; nevertheless, the simultaneous account of the ${ }^{3} \mathrm{H}$ binding $\left|E_{3 \mathrm{H}}\right|$ and of the neutron-deuteron doublet scattering length $a_{2}$ was also either impossible at all or led to unnaturally large coupling constants, quite similar to our finding. In addition, we note that all realistic purely nucleonic potentials combined with standard phenomenological $3 N$ forces fitted to $\left|E_{3^{3}}\right|$ are underpredicting $a_{2}$ by a comparable amount [28].

In some parameter domains we noted a surprising sensitivity for the nucleon analyzing power $A_{y}$ of elastic 
nucleon-deuteron scattering. We therefore created a second group of parametrizations consisting of the $3 N$ potentials ( $\mathrm{U} 1$, $\mathrm{U} 4$, U5). The repulsive term with the parameter $B_{0}$ is left out for them; attraction and, if needed, repulsion are both provided by the $A_{2 \pi}$ and $C_{2 \pi}$ terms. In the $3 N$ potentials U4 and U5 the two parameters have opposite signs. We note also that the $3 N$ potential U1 has $B_{0}=0$, whereas the two other parameters yield attraction; a $3 N$ potential with both parameters $A_{2 \pi}$ and $C_{2 \pi}$ yielding repulsion is unable to account for the ${ }^{3} \mathrm{H}$ binding $\left|E_{3_{\mathrm{H}}}\right|$. None of the parametrizations of the second group (U1, $\mathrm{U} 4, \mathrm{U} 5)$ is able to account for the neutron-deuteron doublet scattering length $a_{2}$, but U5 is roughly adjusted to the neutron analyzing power $A_{y}$ of elastic neutron-deuteron scattering at $10-\mathrm{MeV}$ neutron energy.

Additional modifications with further intermediate parameters appear unnecessary to be accounted in the paper, since the two groups of models for an irreducible $3 \mathrm{~N}$ force suffice to describe the observed trends.

\section{RESULTS}

\section{A. Observations made in the parameter search}

In this subsection, we compare the predictions of interactions with the added irreducible $3 N$ potentials, listed in Table I, for selected three-nucleon scattering observables; we do so separately for the two distinct groups (U1, U2, U3) and (U1, U4, U5). We choose selected observables which best illustrate the effects of the added $3 N$ potentials in either group, desired and undesired, i.e., the neutron analyzing power $A_{y}$ of neutron-deuteron elastic scattering at $10 \mathrm{MeV}$ neutron energy, the differential cross section $d \sigma / d \Omega$ and the deuteron analyzing power $A_{y}(d)$ of proton-deuteron elastic scattering at $135-\mathrm{MeV}$ proton energy, and the differential cross section for neutron-deuteron breakup at $13-\mathrm{MeV}$ neutron laboratory energy in the space star configuration.

First, results for group (U1, U2, U3) are illustrated in Figs. 4 and 5. The general trend is that $3 N$ force effects due to the irreducible U1 are insignificant, even smaller than those obtained from the reducible $3 N$ force contributions due to the explicit $\Delta$-isobar excitation; they improve the agreement with the experimental data [29-32] only slightly. In contrast, the effects due to the $3 N$ potential $\mathrm{U} 3$ are larger than those due to the explicit $\Delta$-isobar excitation, but not beneficial for the description of the data. At $135-\mathrm{MeV}$ proton-deuteron elastic scattering, they move the theoretical predictions into the right direction, but they are far too strong, often dramatically overshooting the discrepancy with data. This somehow uncontrolled behavior is not unexpected, given the individually very strong repulsive and attractive terms in $\mathrm{U} 3$. The results for $\mathrm{U} 2$ are intermediate between those for $\mathrm{U} 1$ and $\mathrm{U} 3$. We conclude that the irreducible $3 N$ potential $\mathrm{U} 1$, being the softest one, appears to make most sense physically, even though it predicts $a_{2}=0.559 \mathrm{fm}$ and we have to give up our ambition to account for the experimental $a_{2}$ value precisely, when choosing U1 for further studies.

Concerning the potential CD Bonn $+\Delta+\mathrm{U} 3$ that is forced to fit the neutron-deuteron doublet scattering length $a_{2}$, we observe another interesting similarity in Fig. 4 with the results

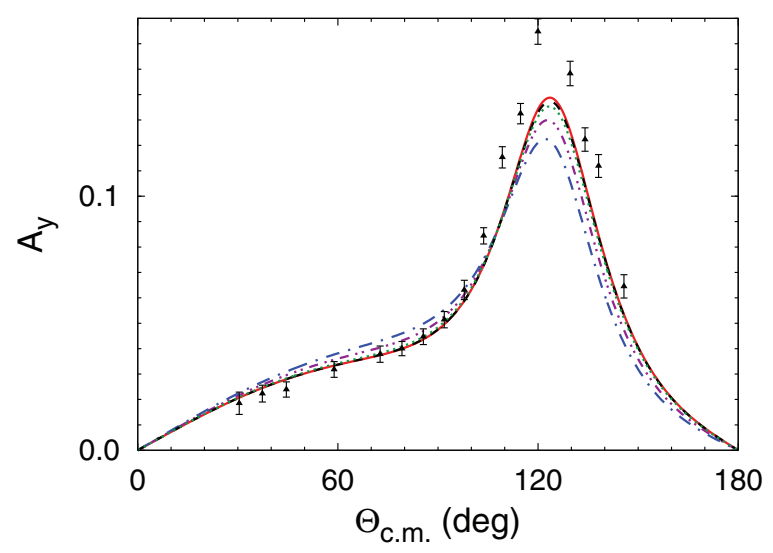

FIG. 4. (Color online) Neutron analyzing power $A_{y}$ for neutrondeuteron elastic scattering at $10-\mathrm{MeV}$ neutron energy as function of the center-of-mass scattering angle $\Theta_{\text {c.m. }}$. Results of CD Bonn (dotted curve), CD Bonn $+\Delta$ (dashed curve), CD Bonn $+\Delta+\mathrm{U} 1$ (solid curve), CD Bonn $+\Delta+\mathrm{U} 2$ (double-dotted-dashed curve), and $\mathrm{CD}$ Bonn $+\Delta+\mathrm{U} 3$ (dotted-dashed curve) are compared with the experimental data from Ref. [29].

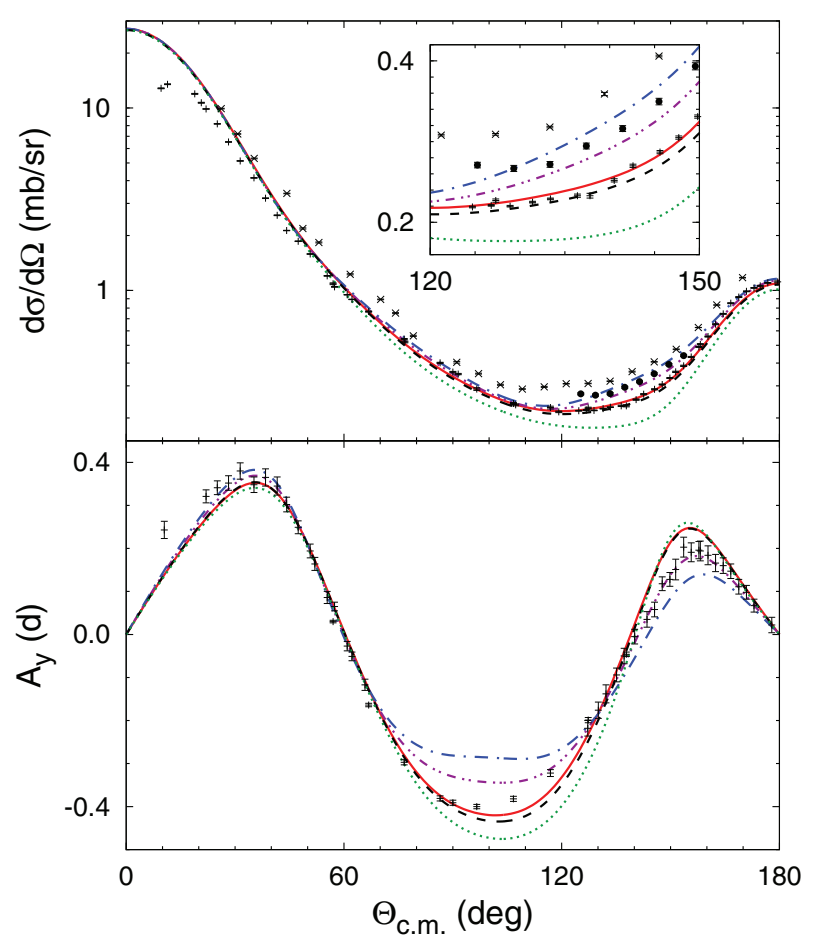

FIG. 5. (Color online) Differential cross section and deuteron vector analyzing power for proton-deuteron elastic scattering at $135-\mathrm{MeV}$ proton energy as function of the center-of-mass scattering angle $\Theta_{\text {c.m. }}$. Curves as in Fig. 4. The theoretical predictions of this figure are obtained omitting the Coulomb repulsion between the protons for reasons of calculational simplicity; at this energy Coulomb does not yield any noticeable effect at angles $\Theta_{\text {c.m. }}>20^{\circ}$ [20]; its inclusion is therefore immaterial for the objectives of this subsection. The experimental data are from Refs. [30] (x), [31] (•), and [32] (+). 


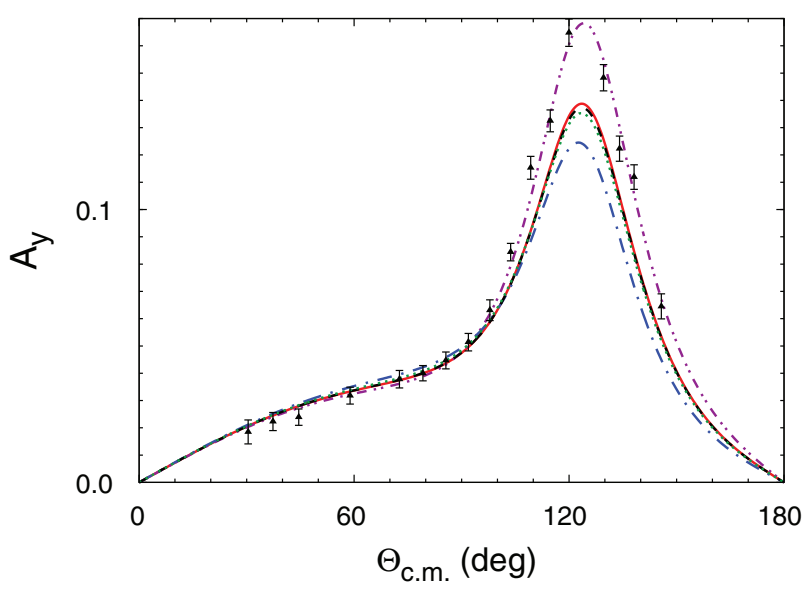

FIG. 6. (Color online) Neutron analyzing power $A_{y}$ for neutrondeuteron elastic scattering at $10-\mathrm{MeV}$ neutron energy as function of the center-of-mass scattering angle $\Theta_{\text {c.m. }}$. Results of CD Bonn (dotted curve), $\mathrm{CD}$ Bonn $+\Delta$ (dashed curve), $\mathrm{CD}$ Bonn $+\Delta+\mathrm{U} 1$ (solid curve), $\mathrm{CD}$ Bonn $+\Delta+\mathrm{U} 4$ (dotted-dashed curve), and CD Bonn $+\Delta+\mathrm{U} 5$ (double-dotted-dashed curve) are compared with the experimental data from Ref. [29].

of Ref. [27], which are obtained from $\chi$ EFT $2 N$ and $3 N$ forces, also fitted to the ${ }^{3} \mathrm{H}$ binding $\left|E_{3^{3} \mathrm{H}}\right|$ and to the neutron-deuteron doublet scattering length $a_{2}$. In addition to the similarity found in Sec. II B, we observe that in both cases, based on completely different dynamic models, the neutron analyzing power $A_{y}$ in neutron-deuteron elastic scattering at $10 \mathrm{MeV}$ is increased at angles $\Theta_{\text {c.m. }}<100^{\circ}$ but decreased $\Theta_{\text {c.m. }}>110^{\circ}$, moving theoretical predictions away from the experimental data. Thus, this additional undesired effect of irreducible $3 \mathrm{~N}$ forces on the analyzing power $A_{y}$ appears to be a general feature of presently available force models that fit $2 N$ data, $\left|E_{3_{3} \mathrm{H}}\right|$, and $a_{2}$ simultaneously and points to a further aspect of the long-standing $A_{y}$ puzzle in low-energy $3 N$ scattering. In Ref. [26] the analyzing power was studied only at $3 \mathrm{MeV}$, but the conclusions are essentially the same, i.e., improving $a_{2}$ increases the discrepancy for $A_{y}$, except when using the Argonne V18 potential supplemented with a particular $3 N$ force of $\chi \mathrm{EFT}$.

Second, the above conjecture on the general difficulty of accounting simultaneously for characteristic three-nucleon data, seen for the potential group (U1, U2, U3), is supported also by our results for the potential group (U1, U4, U5) as shown in Fig. 6. The model CD Bonn $+\Delta+\mathrm{U} 5$ is adjusted to reproduce the experimental data for the neutron analyzing power $A_{y}$ at $10 \mathrm{MeV}$, seemingly overcoming the long-standing $A_{y}$ puzzle. The price for this fit, however, is an increased discrepancy for the neutron-deuteron doublet scattering length $a_{2}$, as can be seen in Table I. The $3 N$ potential U4 that improves $a_{2}$ compared to $\mathrm{U} 1$, increases the discrepancy for $A_{y}$ at the same time, much like $\mathrm{U} 2$ and $\mathrm{U} 3$ do. Thus, again, the simultaneous account of $2 N$ data, $\left|E_{3} \mathrm{H}\right|, a_{2}$, and $A_{y}$ appears to be impossible with the presently available force models. The models CD Bonn $+\Delta+\mathrm{U} 4$ and $\mathrm{CD}$ Bonn $+\Delta+\mathrm{U} 5$, although the latter is successful for low-energy $A_{y}$, contain unusually large parameters $A_{2 \pi}$ and $C_{2 \pi}$, which make the $3 N$ potentials $\mathrm{U} 4$ and U5 as questionable as U3 before. As shown in

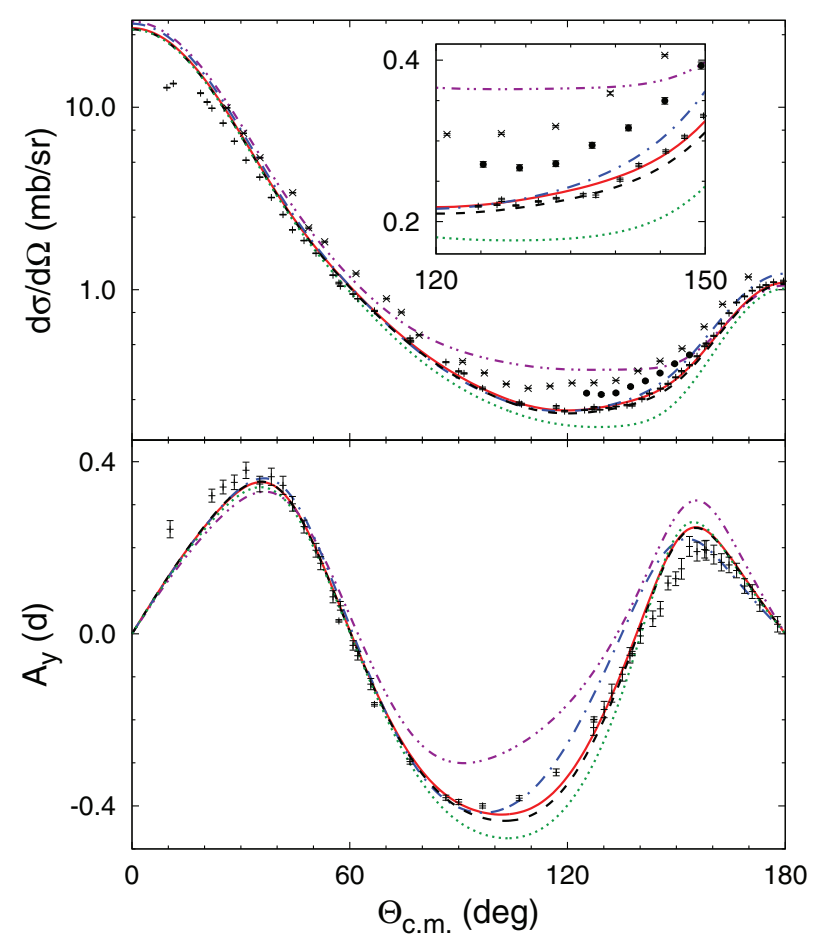

FIG. 7. (Color online) Differential cross section and deuteron vector analyzing power for proton-deuteron elastic scattering at $135-\mathrm{MeV}$ proton energy as function of the center-of-mass scattering angle $\Theta_{\text {c.m. }}$. The theoretical predictions of this figure are obtained omitting the Coulomb force for reasons explained in the caption of Fig. 5. Curves as in Fig. 6. The experimental data are quoted in Fig. 5.

Fig. 7 for proton-deuteron elastic scattering at $135 \mathrm{MeV}$, U5 especially yields a very strong effect that destroys the reasonable agreement with data achieved by the potentials $C D$ Bonn $+\Delta$ or CD Bonn $+\Delta+\mathrm{U} 1$.

Finally, Fig. 8 studies another famous $3 N$ puzzle as bothersome as the $A_{y}$ problem, i.e., the fivefold differential cross section $d^{5} \sigma / d S d \Omega_{1} d \Omega_{2}$ of the nucleon-deuteron breakup at $13 \mathrm{MeV}$ in the space star configuration characterized by the polar and azimuthal angles of two detected nucleons $\left(\theta_{1}, \theta_{2}, \varphi_{2}-\varphi_{1}\right)=\left(50.5^{\circ}, 50.5^{\circ}, 120.0^{\circ}\right)$. This observable is known to be quite insensitive to changes in the Hamiltonian $[33,34]$. Indeed, even the effect of the very strong $3 N$ forces U3 and U5 is insignificant and much smaller than the discrepancy between the predictions and experimental data [35-37]. Figure 8 presents theoretical results for neutrondeuteron scattering; the corresponding theoretical results for proton-deuteron breakup also strongly disagree with the data, despite the inclusion of Coulomb in the calculations; the proton-deuteron results overpredict the data. Thus, none of the considered $3 N$ force models studied by us is able to cure the space star anomaly.

\section{B. Overview on results obtained with the additional $3 N$ potential $\mathrm{U} 1$}

We choose the irreducible $3 N$ potential $\mathrm{U} 1$ as the most reasonable one for a further comparative study of irreducible and reducible contributions to the $3 N$ force; the addition of 


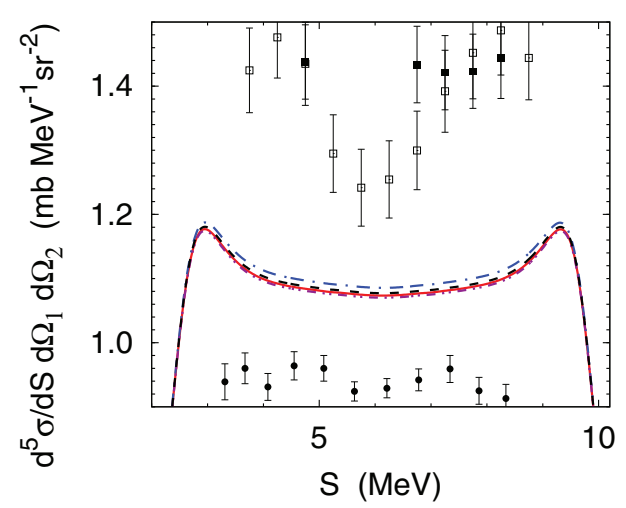

FIG. 8. (Color online) Differential cross section for neutrondeuteron breakup at $13-\mathrm{MeV}$ neutron laboratory energy in the space star configuration as function of the arclength $S$ along the kinematical curve. Results CD Bonn $+\Delta$ (dashed curve), CD Bonn $+\Delta+\mathrm{U} 1$ (solid curve), CD Bonn $+\Delta+\mathrm{U} 3$ (dotted-dashed curve), and $\mathrm{CD}$ Bonn $+\Delta+\mathrm{U} 5$ (double-dotted-dashed curve) are compared with the experimental data from Refs. [35,36] (open and full squares). The proton-deuteron data from Ref. [37] (full circles) are also shown.

U1 accounts for the binding of ${ }^{3} \mathrm{H}$ by construction, but it also yields the ${ }^{3} \mathrm{H}-{ }^{3} \mathrm{He}$ mass difference perfectly, i.e., the binding of ${ }^{3} \mathrm{He}$ becomes $7.718 \mathrm{MeV}$, its experimental value also being $7.718 \mathrm{MeV}$. In this subsection for a wide range of $3 \mathrm{~N}$ scattering observables we compare the predictions of the potentials $\mathrm{CD}$ Bonn without any $3 N$ force, $\mathrm{CD}$ Bonn $+\Delta$ with reducible contributions to the $3 N$ force, and of CD Bonn $+\Delta+\mathrm{U} 1$ with reducible and irreducible contributions to the $3 N$ force.

In Fig. 9 we show the differential cross section and various spin observables for elastic proton-deuteron scattering at $135 \mathrm{MeV}$, in Fig. 10 the differential cross section and nucleon-to-nucleon spin transfer coefficients for elastic proton-deuteron scattering at $250 \mathrm{MeV}$, and in Fig. 11 the fivefold differential cross section and nucleon analyzing powers for proton-deuteron breakup at $135 \mathrm{MeV}$. In contrast to Sec. III A, we carry out the calculations for proton-deuteron scattering fully and include the proton-proton Coulomb force using the method of screening and renormalization [18-21], though we do not show the explicit Coulomb effects separately in the plots. In elastic scattering at these energies the Coulomb effect is irrelevant, except for small angles [20]; it is seen in Figs. 9 and Fig. 10 as wiggles in all observables below $\Theta_{\text {c.m. }}<10^{\circ}$. In breakup the Coulomb effect is most visible in configurations with low relative proton-proton energy [21], mostly affecting the differential cross section. For example, in the configuration $\left(28^{\circ}, 28^{\circ}, 20^{\circ}\right)$ of Fig. 11 the proton-proton Coulomb force reduces the cross section at the peak by about $25 \%$.

The reducible contributions to the $3 N$ force, i.e., the explicit $\Delta$-isobar excitation, create the largest effects in the studied observables. The additional effects of the irreducible $3 N$ force $\mathrm{U} 1$ are minor even at higher energies, and they do not destroy any achieved account of the experimental data. In proton-deuteron elastic scattering, the reducible and irreducible $3 N$-force contributions move the predictions quite often into the same direction such that the inclusion of

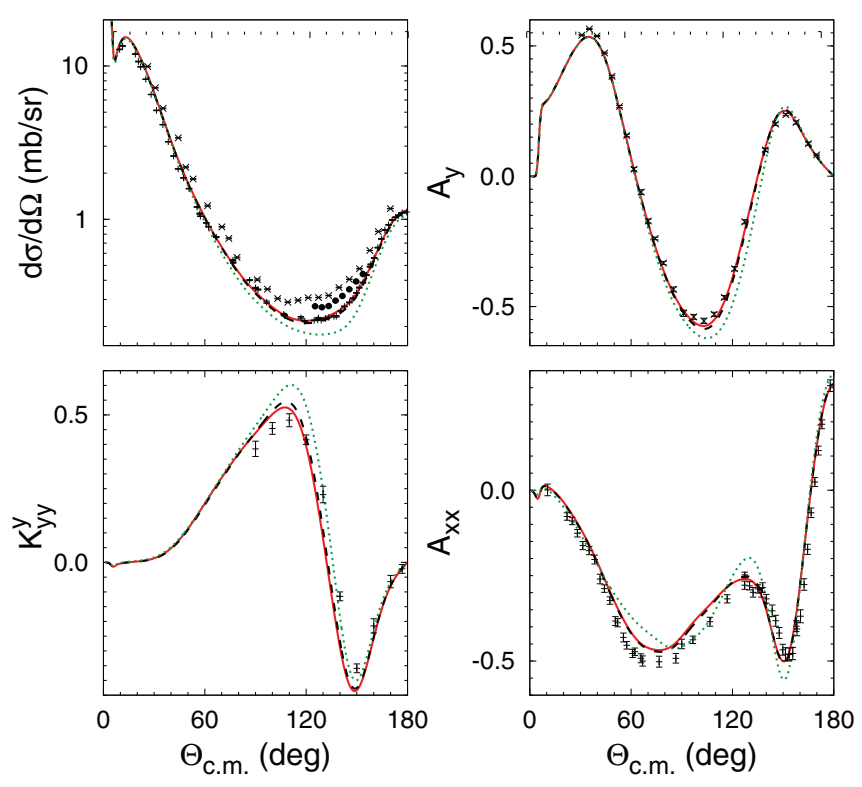

FIG. 9. (Color online) Differential cross section, proton analyzing power $A_{y}$, deuteron-to-proton spin-transfer coefficient $K_{y y}^{y}$, and deuteron analyzing power $A_{x x}$ for proton-deuteron elastic scattering at $135-\mathrm{MeV}$ proton energy. Results based on the potentials CD Bonn (dotted curve), CD Bonn $+\Delta$ (dashed curve), and CD Bonn $+\Delta+\mathrm{U} 1$ (solid curve) are shown. The experimental data as in Fig. 5.

irreducible $3 N$ force $\mathrm{U} 1$ even slightly improves the description of the experimental data. The most prominent examples are the differential cross section around the minimum and at backward angles, the nucleon analyzing power $A_{y}$ at $135 \mathrm{MeV}$ around the minimum, and the nucleon-to-nucleon spin transfer coefficient

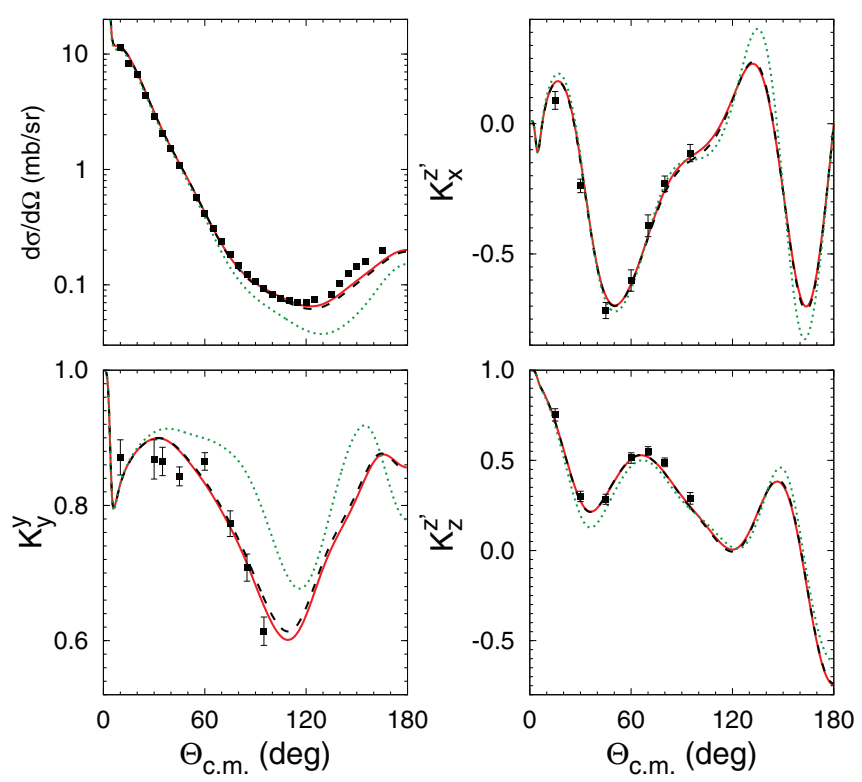

FIG. 10. (Color online) Differential cross section and nucleonto-nucleon spin-transfer coefficients for proton-deuteron elastic scattering at $250-\mathrm{MeV}$ proton energy. Note that this energy is slightly above the $\pi$-production threshold but remains well below the $\Delta$-production threshold. Curves as in Fig. 9. The experimental data are from Ref. [38]. 


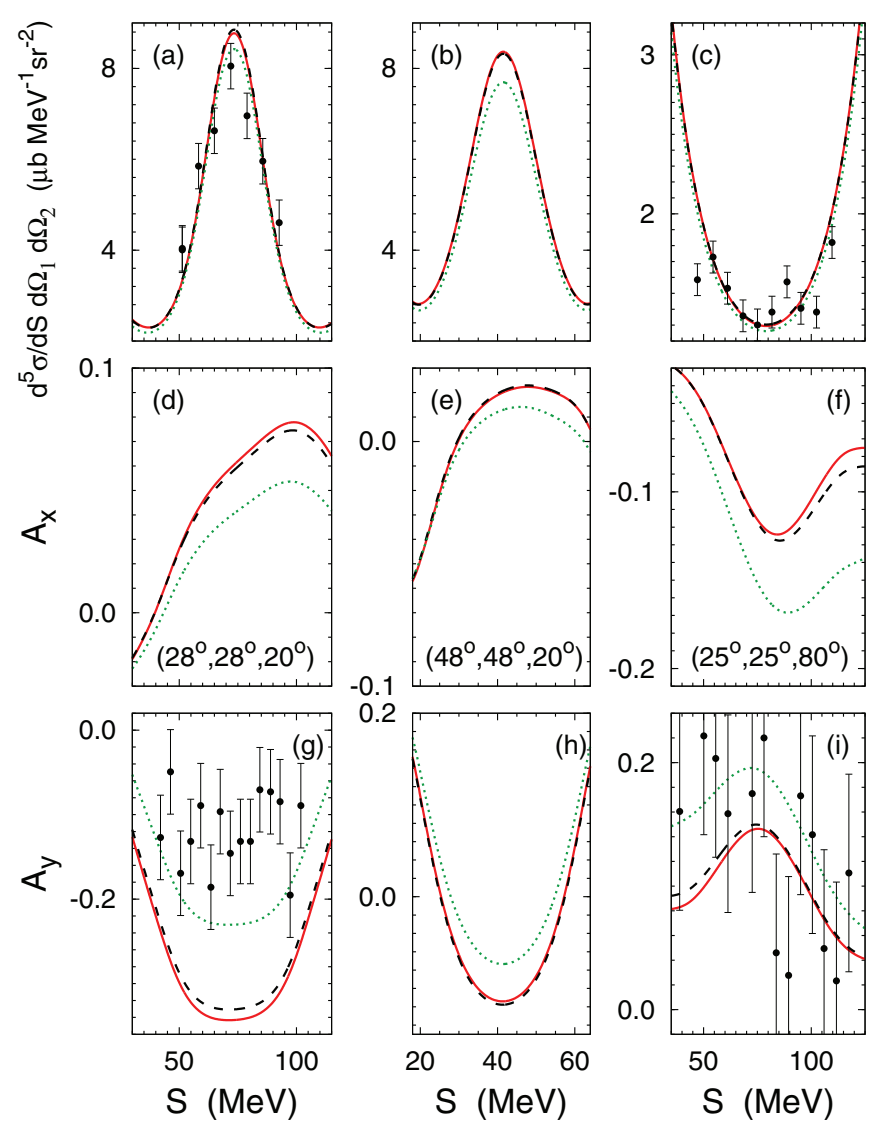

FIG. 11. (Color online) Differential cross section and proton analyzing powers $A_{x}$ and $A_{y}$ for proton-deuteron breakup at $135-\mathrm{MeV}$ proton energy. Results for three kinematical configurations $\left(28^{\circ}, 28^{\circ}, 20^{\circ}\right),\left(48^{\circ}, 48^{\circ}, 20^{\circ}\right)$, and $\left(25^{\circ}, 25^{\circ}, 80^{\circ}\right)$ are given from left and to right, respectively. Curves as in Fig. 9. The experimental data are from Ref. [39].

$K_{y}^{y}$ at $250 \mathrm{MeV}$, as well as the deuteron vector analyzing power $A_{y}(d)$ at $135 \mathrm{MeV}$ shown in Fig. 5. In the breakup reaction the reducible and irreducible $3 N$ forces may have effects of the same or of opposite signs as illustrated in Fig. 11, depending on the kinematical configuration and depending on the specific observable. In the considered example the Coulomb repulsion between the protons is important for the achieved gross agreement with the cross-section data, but neither $3 N$ force contribution is beneficial in accounting for the experimental $A_{y}$ data of Ref. [39], especially in the $\left(28^{\circ}, 28^{\circ}, 20^{\circ}\right)$ configuration.

\section{CONCLUSIONS}

In the present work we describe the properties of the threenucleon system, i.e., the bound state and the nucleon-deuteron elastic scattering and breakup below the $\pi$-production threshold, in an extended Hilbert space with a coupled-channels Hamiltonian containing irreducible two- and three-baryon potentials. The extension of the Hilbert space is due to a sector in which one nucleon is turned into a $\Delta$ isobar. The Hamiltonian couples both sectors of the Hilbert space. The $\Delta$ isobar makes important, reducible, easily calculable contributions to the $2 N$ and $3 N$ interactions; the most prominent contribution is the Fujita-Miyazawa mechanism to the $3 N$ interaction, even enriched by the $\rho$ meson contributions. The irreducible three-baryon potential is constrained to a purely nucleonic one. The simplification of the additional Hilbert sector with a single $\Delta$ isobar only and the constraint of the irreducible three-baryon potential to a nucleonic one only are well motivated.

We prove that calculations in such an extended Hilbert space with such an extended Hamiltonian can be computationally done with high precision. The computational technology can, in future, be extended to other and conceptually better founded forces, e.g., to those possibly provided by $\chi \mathrm{EFT}$ with an explicit $\Delta$ isobar [5,6], kept in a quantum-mechanical Hamiltonian still to be derived. Thus, the technical objective of the paper is reached.

We explored the effect of irreducible $3 N$ potentials of the Urbana IX type on top of the coupled-channels two-baryon potential $C D$ Bonn $+\Delta$. We found that the simultaneous precise account of the ${ }^{3} \mathrm{H}$ binding energy $\left|E_{{ }^{3} \mathrm{H}}\right|$ and of the neutrondeuteron doublet scattering length $a_{2}$ is extremely difficult to achieve. This finding may appear to be due to our special theoretical framework. But this is a finding encountered also in Ref. [27] using the framework of entirely different nuclear forces of $\chi$ EFT and in Ref. [26] employing the Argonne V18 potential combined with variations of the Urbana IX and Tucson-Melbourne $3 \mathrm{~N}$ forces. Thus, this finding is surprisingly more general than expected and may constitute another theoretical puzzle in the three-nucleon system. Furthermore, the simultaneous account of $2 N$ data, of the ${ }^{3} \mathrm{H}$ binding $\left|E_{3} \mathrm{H}\right|$, of the neutron-deuteron doublet scattering length $a_{2}$ and of the low-energy nucleon analyzing power $A_{y}$ appears to be even more difficult to achieve, and that problem also calls for new terms in the Hamiltonian, especially of the $3 \mathrm{~N}$ nature.

We employed the irreducible $3 N$ potential U1 of Table I as dynamic basis for the description of three-nucleon binding and scattering, together with the coupled-channels two-baryon potential CD Bonn $+\Delta$. The $3 N$ potential U1 provides the missing contribution to the three-nucleon binding and it is weak. This is wanted, but we have to give up our original goal of also accounting for the neutron-deuteron doublet scattering length $a_{2}$ precisely. Nevertheless, the results for three-nucleon scattering at higher energies are satisfactory, since the broad range of the successful account of experimental data without an irreducible $3 N$ potential is preserved and often even slightly improved. However, the well-known puzzles of low-energy three-nucleon scattering, i.e., the nucleon analyzing power $A_{y}$ of elastic nucleon-deuteron scattering below $20 \mathrm{MeV}$ nucleon lab energy and the nucleon-deuteron breakup in the space star kinematics stay unresolved. That is a disappointing aspect of the present results but not unexpected given the previous studies [26,27,33,34].

\section{ACKNOWLEDGMENTS}

A.D. thanks the Leibniz University Hannover for the hospitality during the completion of this work. The work of A.D. is partially supported by the Research Council of Lithuania under Contract No. SF4-007/VYK-015. 
[1] R. Machleidt, K. Holinde, and C. Elster, Phys. Rep. 149, 1 (1987).

[2] E. Epelbaum, Prog. Part. Nucl. Phys. 57, 654 (2006).

[3] E. Epelbaum, H.-W. Hammer, and U.-G. Meißner, Rev. Mod. Phys. 81, 1773 (2009).

[4] R. Machleidt and D. R. Entem, Phys. Rep. 503, 1 (2011).

[5] H. Krebs, E. Epelbaum, and U.-G. Meißner, Eur. Phys. J. A 32, 127 (2007).

[6] E. Epelbaum, H. Krebs, and U.-G. Meißner, Nucl. Phys. A 806, 65 (2008).

[7] A. Deltuva, R. Machleidt, and P. U. Sauer, Phys. Rev. C 68, 024005 (2003).

[8] J. Fujita and H. Miyazawa, Prog. Theor. Phys. 17, 360 (1957).

[9] H. Krebs, A. Gasparyan, and E. Epelbaum, Phys. Rev. C 87, 054007 (2013).

[10] S. C. Pieper, V. R. Pandharipande, R. B. Wiringa, and J. Carlson, Phys. Rev. C 64, 014001 (2001).

[11] S. Nemoto, K. Chmielewski, J. Haidenbauer, S. Oryu, P. U. Sauer, and N. W. Schellingerhout, Few-Body Systems 24, 213 (1998).

[12] A. Deltuva, K. Chmielewski, and P. U. Sauer, Phys. Rev. C 67, 034001 (2003).

[13] A. Deltuva, Phys. Rev. C 80, 064002 (2009).

[14] A. Stadler and P. U. Sauer, Phys. Rev. C 46, 64 (1992).

[15] E. O. Alt, P. Grassberger, and W. Sandhas, Nucl. Phys. B 2, 167 (1967).

[16] B. S. Pudliner, V. R. Pandharipande, J. Carlson, S. C. Pieper, and R. B. Wiringa, Phys. Rev. C 56, 1720 (1997).

[17] R. Lazauskas, Ph.D. thesis, Université Joseph Fourier, Grenoble, 2003 [http://tel.ccsd.cnrs.fr/documents/archives0 /00/00/41/78/]

[18] J. R. Taylor, Nuovo Cimento B 23, 313 (1974); M. D. Semon and J. R. Taylor, Nuovo Cimento A 26, 48 (1975).
[19] E. O. Alt and W. Sandhas, Phys. Rev. C 21, 1733 (1980).

[20] A. Deltuva, A. C. Fonseca, and P. U. Sauer, Phys. Rev. C 71, 054005 (2005).

[21] A. Deltuva, A. C. Fonseca, and P. U. Sauer, Phys. Rev. C 72, 054004 (2005).

[22] R. B. Wiringa, V. G. J. Stoks, and R. Schiavilla, Phys. Rev. C 51, 38 (1995).

[23] S. A. Coon, M. D. Scadron, P. C. McNamee, B. R. Barrett, D. W. E. Blatt, and B. H. J. McKellar, Nucl. Phys. A 317, 242 (1979).

[24] W. Manzke and M. Gari, Nucl. Phys. A 312, 457 (1978).

[25] W. Dilg, L. Koester, and W. Nistler, Phys. Lett. B 36, 208 (1971).

[26] A. Kievsky, M. Viviani, L. Girlanda, and L. E. Marcucci, Phys. Rev. C 81, 044003 (2010).

[27] J. Golak et al., Eur. Phys. J. A 50, 177 (2014).

[28] H. Witała, A. Nogga, H. Kamada, W. Glöckle, J. Golak, and R. Skibiński, Phys. Rev. C 68, 034002 (2003).

[29] C. R. Howell et al., Few-Body Systems 2, 19 (1987).

[30] K. Ermisch et al., Phys. Rev. C 71, 064004 (2005).

[31] A. Ramazani-Moghaddam-Arani et al., Phys. Rev. C 78, 014006 (2008).

[32] K. Sekiguchi et al., Phys. Rev. C 65, 034003 (2002).

[33] H. Witała and W. Glöckle, J. Phys. G 37, 064003 (2010).

[34] S. Ishikawa, Phys. Rev. C 80, 054002 (2009).

[35] J. Strate et al., Nucl. Phys. A 501, 51 (1989).

[36] H. R. Setze et al., Phys. Rev. C 71, 034006 (2005).

[37] G. Rauprich, S. Lemaitre, P. Niessen, K. R. Nyga, R. Reckenfelderbäumer, L. Sydow, H. Paetz gen. Schieck, H. Witała, and W. Glöckle, Nucl. Phys. A 535, 313 (1991).

[38] K. Hatanaka et al., Phys. Rev. C 66, 044002 (2002).

[39] M. Eslami-Kalantari et al., Eur. Phys. J. Web Conf. 3, 05010 (2010). 